\title{
Sodium Butyrate Protects Against Ethanol-Induced Toxicity in SH-SY5Y Cell Line
}

\author{
Bruk Getachew ${ }^{1} \cdot$ Antonei B. Csoka $^{2} \cdot$ Allison R. Garden ${ }^{1} \cdot$ Robert L. Copeland $^{1} \cdot$ Yousef Tizabi $^{1} \mathbb{C}$
}

Received: 15 August 2021 / Revised: 13 September 2021 / Accepted: 14 September 2021 / Published online: 23 September 2021

(c) The Author(s), under exclusive licence to Springer Science+Business Media, LLC, part of Springer Nature 2021

\begin{abstract}
Alcohol use disorder (AUD), brought about by excessive alcohol use, is associated with damages to several organs including the brain. Chronic excessive use of alcohol can compromise intestinal integrity, leading to changes in gut microbiota (GM) composition known as dysbiosis. Dysbiosis, by disruption of the gut-brain axis (GBA), further exacerbates the deleterious effects of alcohol. One of the fermentation by-products of GM is butyrate (BUT), a short-chain fatty acid (SCFA) that plays an important role in maintaining homeostasis of the GBA. Alcohol metabolism results in formation of acetaldehyde, a highly reactive compound that reacts with dopamine in the brain to form toxic adducts such as salsolinol. Recent studies indicate potential neuro-protective effects of BUT against various toxicants including salsolinol. Here, we sought to investigate whether BUT can also protect against alcohol toxicity. Pretreatment of neuroblastoma-derived SH-SY5Y cells with $500 \mathrm{mM}$ ethanol (ETOH) for $24 \mathrm{~h}$ resulted in approximately $40 \%$ reduction in cell viability, which was totally blocked by $10 \mu \mathrm{M}$ of either BUT or AR 420,626 (AR), a selective fatty acid 3 receptor (FA3R) agonist. The neuro-protective effects of both BUT and AR were significantly (80\%) attenuated by beta-hydroxy butyrate (BHB), a selective FA3R antagonist. Interestingly, combination of BUT and AR resulted in synergistic protection against ETOH, which was totally blocked by BHB. These findings suggest potential utility of butyrate and/or FA3R agonists against ETOH-induced toxicity.
\end{abstract}

Keywords Alcohol $\cdot$ Butyrate $\cdot$ Short-chain fatty acids (SCFAs) $\cdot$ Gut microbiota $\cdot$ Fatty acid 3 receptor (FA3R) $\cdot$ Betahydroxy butyrate

\section{Introduction}

Chronic excessive alcohol (CEA) use could lead to alcohol used disorder (AUD), a problematic pattern of alcohol use with an impaired ability to stop/control alcohol use despite adverse social, occupational, or heath consequences (Koob 2021). CEA can cause neuronal loss by inducing neurotoxicity as well as inhibiting neurogenesis. This leads to significant volume loss in cortical and subcortical brain structures and shrinkage in both gray and white matters. These structural changes along with impairment in neuronal signaling and glial dysfunction result in short-term memory

Yousef Tizabi

ytizabi@howard.edu

1 Department of Pharmacology, Howard University College of Medicine, 520 W Street NW, Washington, DC 20059, USA

2 Department of Anatomy, Howard University College of Medicine, Washington, DC, USA loss and other behavioral abnormalities such as weakness, ataxia, motor speech disorder, diminished executive functioning, anxiety, and negative emotions (Tizabi et al. 2021b).

Recent development in the gut-brain axis (GBA) has confirmed a bidirectional and intimate cross talk between gut microbiome and the brain. Thus, the importance of normal intestinal colonization of the gut, starting at neonatal period and continuing throughout life, in critical physiological functions has been well established. Gut microbiome plays an essential role in food digestion, xenobiotic metabolism, and regulation of innate and adaptive immunological processes (Bishehsari et al. 2017; Cryan et al. 2019). Indeed, dysbiosis, a term representing a loss or reduction of beneficial bacteria and accumulation of pathogenic ones (Belizário and Faintuch 2018), has been associated with dysregulation of food intake and energy homeostasis (Romaní-Pérez et al. 2021; Woźniak et al. 2021) and implicated in a myriad of central nervous system (CNS) disorders such as anxiety and depression (Cryan et al. 2019; Knudsen et al. 2021; Poluektova et al. 2021), 
stroke (Yamashiro et al. 2021), multiple sclerosis (Ullah et al. 2021), Parkinson's disease (Tizabi et al. 2021a), autism (Kong et al. 2021), schizophrenia (Kelly et al. 2021), aging ( $\mathrm{Li}$ et al. 2021; van Krimpen et al. 2021), cognitive dysfunction (Cryan et al. 2019; Cowan and Cryan 2021; Li et al. 2021), Alzheimer's disease (Giovannini et al. 2021; Jiang et al. 2021), and even substance abuse (Meckel and Kiraly 2019; García-Cabrerizo et al. 2021).

Dysbiosis due to alcohol abuse may be a contributing factor to AUD (Engen et al. 2015; Bishehsari et al. 2017). Thus, alcohol-induced changes in the gut microbiota may contribute not only to development of alcoholic liver disease, but also to a variety of CNS disorders (Engen et al. 2015; García-Cabrerizo et al. 2021). Indeed, it has been suggested that use of probiotic might be of potential use in prevention and treatment of alcohol-associated pathologies (Engen et al. 2015; Lowe et al. 2018; Rodriguez-Gonzalez and Orio 2020; Carbia et al. 2021; Gupta et al. 2021). In this regard, it was recently reported that butyrate (BUT) ameliorates alcoholic fatty liver disease in mice (Zhang et al. 2021).

Butyrate is a short-chain fatty acid (FA) that acts as an energy source for colonic epithelial cells. It has antiinflammatory, enteroendocrine, and epigenetic effects and can also affect the brain function (Cantu-Jungles et al. 2019). The postulated mechanism of action of BUT includes its interaction with fatty acid receptor 3 (FA3R) which is a G protein coupled receptor, as well as inhibition of histone deacetylase (HDAC) (Cantu-Jungles et al. 2019; FalomirLockhart et al. 2019). Recently, our group reported protective effects of BUT against salsolinol-induced toxicity in neuroblastoma-derived SH-SY5Y cells (Getachew et al. 2020). The protective effects of BUT were mimicked by another FA3R agonist (AR), and both BUT and AR could be blocked by the FA3R antagonist, beta-hydroxy butyrate (BHB) (Kimura et al. 2011; Ulven 2012; Inoue et al. 2014; Getachew et al. 2020). Since salsolinol is produced via condensation of dopamine with aldehydes and was shown lately to be present in the ventral tegmental area of rats following oral alcohol administration (Bassareo et al. 2021), we undertook this study to determine whether BUT and/ or AR could also protect against alcohol-induce toxicity in SH-SY5Y cells.

\section{Materials and Methods}

Ethanol (ETOH, 100\%) was obtained from EMD Chemicals Inc. (Gibbstown, NJ). Butyrate, beta-hydroxy butyrate (BHB), a selective FA3R antagonist (Kimura et al. 2011; Ulven 2012; Inoue et al. 2014), and other analytical reagents including 3,(4,5-dimethylthiazol-2-yl)-2,5 diphenyltetrazolium bromide (MTT) assay kit were purchased from Sigma Chemical Company (Sigma-Aldrich, St. Louis, MO). AR 420,626
(AR), chemical name: $N$-(2,5-dichlorophenyl)-4-(furan2-yl)-2-methyl-5-oxo-1,4,5,6,7,8-hexahydroquinoline3-carboxamide, a FA3 receptor agonist (Hudson et al. 2014; Bolognini et al. 2016; Kaji et al. 2018), was purchased form Bio Techne Corp-Tocris (Minneapolis, MN). The SH-SY5Y human neuroblastoma cell line was purchased from American Type Culture Collection (ATCC, Manassas, VA).

As detailed previously (Getachew et al. 2018, 2020), SH-SY5Y cells were cultured in a 1:1 mixture of Dulbecco's modified Eagle's medium (DMEM) and Ham's F12 supplemented with $10 \%$ fetal bovine serum, penicillin/ streptomycin $(100 \mathrm{IU} / \mathrm{ml})$, and gentamicin $(50 \mu \mathrm{g} / \mathrm{ml})$ at $37{ }^{\circ} \mathrm{C}$ in $95 \% \mathrm{O}_{2} / 5 \% \mathrm{CO}_{2}$ humidified incubator. The cells (un-differentiated) were trypsinized when confluent and plated in 96-well plates $\left(1.2 \times 10^{4}\right.$ cells/well $)$. Cells were allowed to adhere to bottom surface for $24 \mathrm{~h}$. Then, fresh media containing ethanol $(500 \mathrm{mM})$ or various concentrations of BUT or AR with and without FA3R antagonist were added to the carefully aspirated wells. The concentration of ethanol was chosen based on our previous experiments and the fact that such concentration results in approximately $40 \%$ toxicity in SH-SY5Y cells (Getachew et al. 2018). Butyrate or AR was added $1 \mathrm{~h}$ prior to ETOH, and FA3R antagonist, in turn, was added $1 \mathrm{~h}$ prior to BUT or AR. In combination studies, both BUT and AR were added simultaneously $1 \mathrm{~h}$ prior to ETOH. In all cases, the control group consisted of cells that were maintained in media alone and without any drug treatment. All treatments were carried out for $24 \mathrm{~h}$, and the effects on cell viability were determined following the 24-h incubation. Each treatment group consisted of 6 replicates, and a minimum of 4 assays were conducted for each experimental manipulation.

Cell viability was determined by MTT colorimetric assay according to the manufacturer's protocol as described previously (Getachew et al. 2018, 2020). Briefly, the yellow MTT tetrazolium salt $(0.5 \mathrm{mg} / \mathrm{ml})$ was dissolved in phosphatebuffered saline (PBS) with $10 \mathrm{mM}$ (4-(2-hydroxyethyl)1-piperazineethanesulfonic acid (HEPES). Thirty microliters of MTT was added to each well and incubated for $3 \mathrm{~h}$ at $37^{\circ} \mathrm{C}$. The live cells cause a reduction of the yellow salt to insoluble purple formazan crystals. The wells were then carefully aspirated, and $50 \mu \mathrm{l}$ of dimethyl sulfoxide (DMSO) was added to the wells to solubilize the crystals; the plates were then placed in a shaker for an hour and read spectrophotometrically at $570 \mathrm{~nm}$ with a background of $630 \mathrm{~nm}$ in a plate reader. Cell viability was determined by subtracting the test results from the background and is presented as a percentage of the control.

Data is expressed as mean \pm standard error of the mean (SEM). Statistical differences within and between treatment groups were determined by one-way analysis of variance (ANOVA) followed by post hoc Newman-Keuls Multiple comparison test, where $p<0.05$ was considered statistically 
significant. Data were analyzed using GraphPad Prism 9.2 (GraphPad Software, Inc., San Diego, CA).

\section{Results}

Figure 1 depicts the effect of various concentrations of BUT $(0.01-10 \mu \mathrm{M})$ against ETOH-induced toxicity in SHSY5Y cells. For ETOH, we used a concentration of $500 \mathrm{mM}$ because we have consistently observed about $40 \%$ toxicity during the 24-h exposure with this concentration (Getachew et al. 2018). As seen, there was a concentration-dependent protection by BUT against ETOH toxicity with full protection at $10 \mu \mathrm{M}[F(5,24)=14.8, p<0.01]$. Butyrate by itself, at any concentration, did not affect the cell viability (data not shown).

Figure 2 depicts the effect of various concentrations of AR $(0.01-10 \mu \mathrm{M})$ against ETOH-induced toxicity. Here also, there was a concentration-dependent protection by AR against ETOH-induced toxicity. Full protection was achieved at $10 \mu \mathrm{M}$ AR $[F(5,24)=13.6, p<0.01]$. AR by itself, at any concentration, did not affect the cell viability (data not shown).

Figure 3 depicts the effect of various concentrations of FA3R antagonist (BHB) on protective effects of BUT against ETOH-induced toxicity. We used the highest concentration of BUT $(10 \mu \mathrm{M})$ as this concentration was fully protective against ETOH toxicity. As seen, there was a concentration-dependent attenuation of BUT effect by BHB $[F(7,32)=16.4, p<0.01]$. The highest concentration of BHB $(60 \mu \mathrm{M})$ reduced the protective effects of BUT by approximately $80 \%(p<0.01)$. BHB did not have any effect of its own on cell viability at any of the concentrations used (data not shown). We did not try any higher BHB concentration, because at $80 \mu \mathrm{M}$, it caused some of its own toxicity.

Figure 4 depicts the effect of various concentrations of BHB on protective effects of AR against ETOH-induced toxicity. We used the highest concentration of AR $(10 \mu \mathrm{M})$ as this concentration was fully protective against ETOH-induced toxicity. As shown, there was a concentration-dependent attenuation of AR protection by BHB $[F(7,32)=14.5$, $p<0.01]$. In this case also, the highest concentration of BHB $(60 \mu \mathrm{M})$ reduced the protective effects of AR by approximately $80 \%(p<0.01)$.

Figure 5 depicts the effect of combination of low concentrations of BUT and AR. Whereas neither butyrate nor AR at $0.01 \mu \mathrm{M}$ concentration had any protective effect against ETOH toxicity, the combination of the two resulted in approximately $50 \%$ protection $[F(9,42)=12.1,(p<0.01)]$. The combination of $0.1 \mu \mathrm{M}$ BUT and $0.1 \mu \mathrm{M}$ AR, where

\section{Butyrate (BUT) protection against ethanol (ETOH) toxicity}

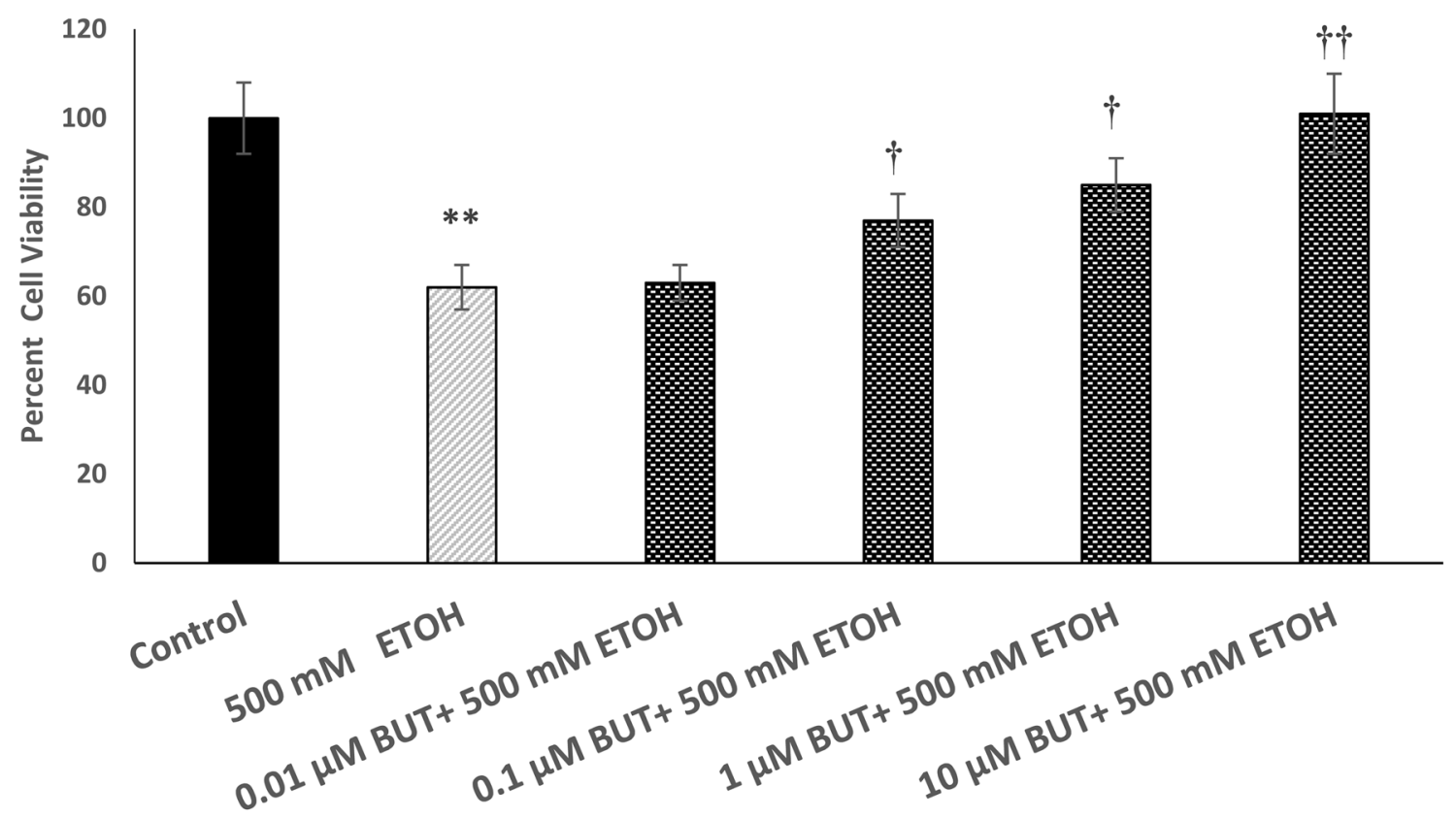

Fig. 1 Effect of various concentrations of butyrate (BUT) against ethanol (ETOH)-induced toxicity. Cells were treated with ETOH with and without BUT for $24 \mathrm{~h}$, and cell viability was determined by
MTT. BUT was added $1 \mathrm{~h}$ before ETOH. Values are mean \pm SEM. ${ }^{* *} p<0.01$ compared to control, ${ }^{\dagger} p<0.5,{ }^{\dagger \dagger} p<0.01$ compared to ETOH. $N=4$ per treatment 


\section{AR protection against ethanol (ETOH) toxicity}

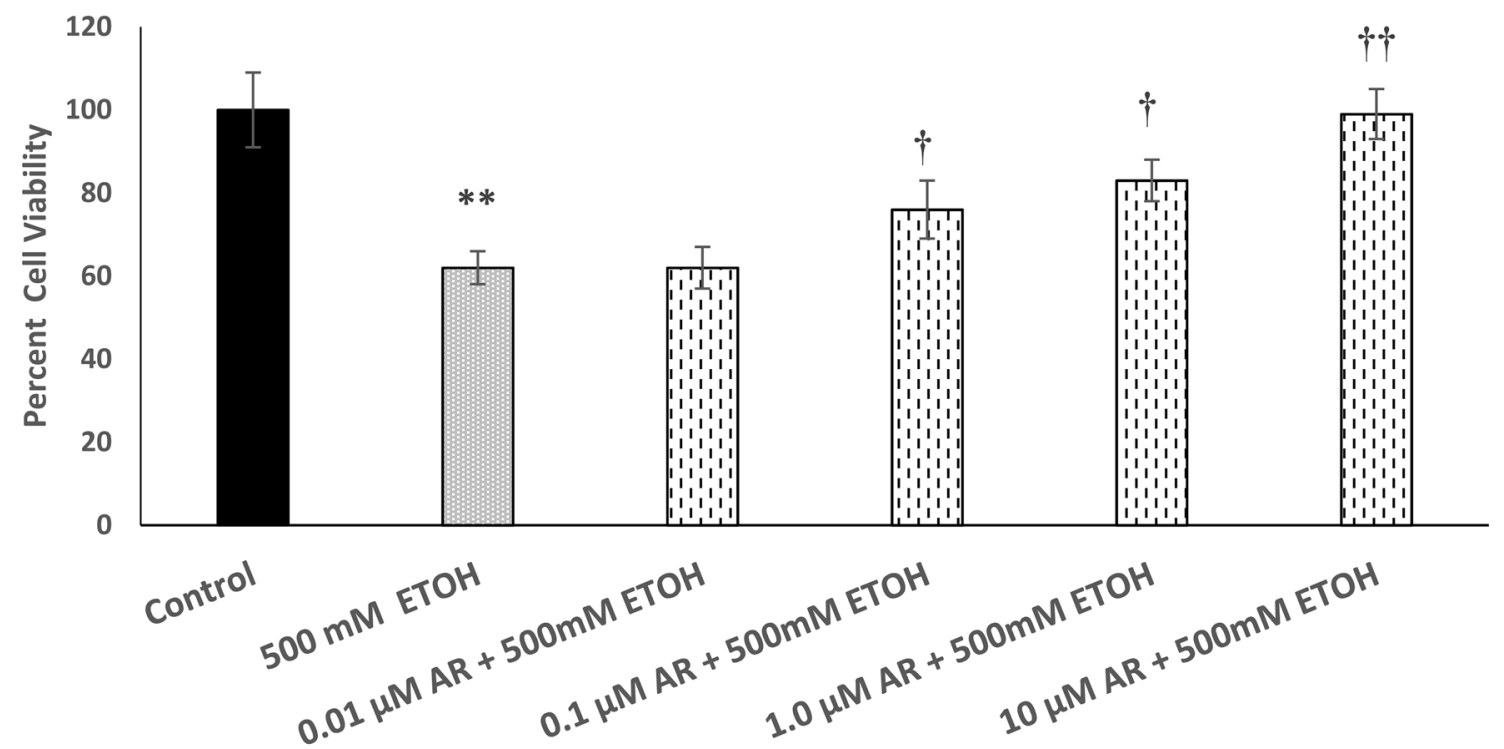

Fig. 2 Effect of various concentrations of AR, a selective FA3R agonist, against ethanol (ETOH)-induced toxicity. Cells were treated with ETOH with and without AR for $24 \mathrm{~h}$, and cell viability was determined by MTT.

each had approximately $24 \%$ protection, resulted in complete protection against ETOH $[F(9,42)=12.1,(p<0.01)]$. Moreover, the effects of the combination treatments at
AR was added $1 \mathrm{~h}$ before ETOH. Values are mean \pm SEM. $* * p<0.01$ compared to control, ${ }^{\dagger} p<0.5,{ }^{\dagger \dagger} p<0.01$ compared to ETOH. $N=4$ per treatment

either concentration levels were totally blocked with the highest concentration of BHB $(60 \mu \mathrm{M})[F(9,42)=12.1$, $(p<0.01)]$.

\section{BHB block of BUT's protective effects}

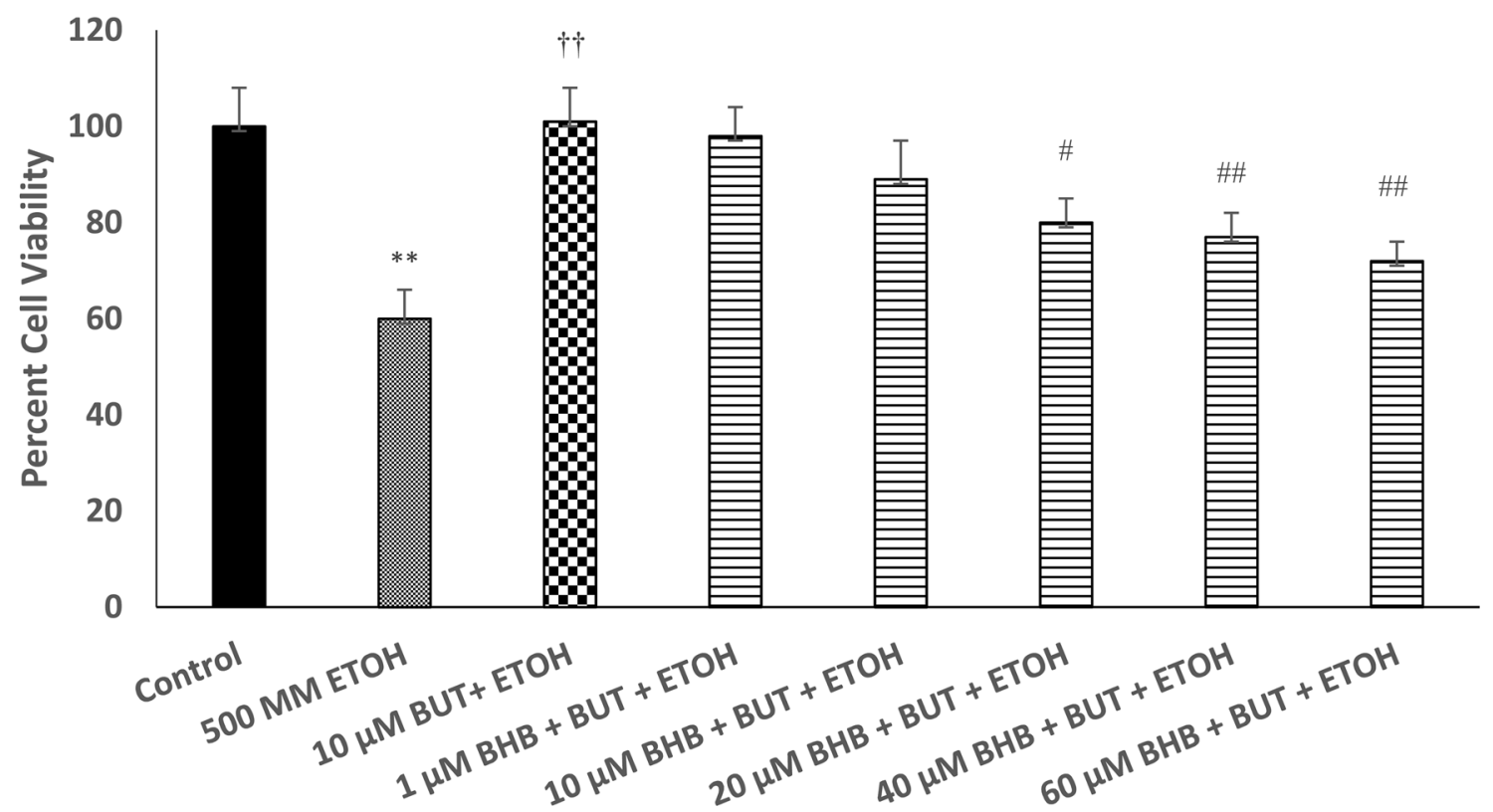

Fig. 3 Effect of various concentrations of beta-hydroxy butyrate (BHB), a FA3R antagonist, on protective effects of butyrate (BUT) against ethanol (ETOH)-induced toxicity. Cells were treated with ETOH, BUT, and BHB for $24 \mathrm{~h}$ and cell viability was determined by MTT. BHB was added $1 \mathrm{~h}$ before BUT, which was added $1 \mathrm{~h}$ before ETOH. Values are mean \pm SEM. $* * p<0.01$ compared to control. ${ }^{\dagger} p<0.01$ compared to $\mathrm{ETOH},{ }^{\#} p<0.05,{ }^{\#} p<0.01$ compared to BUT + ETOH. $N=4$ per treatment 


\section{BHB block of AR's protective effects}

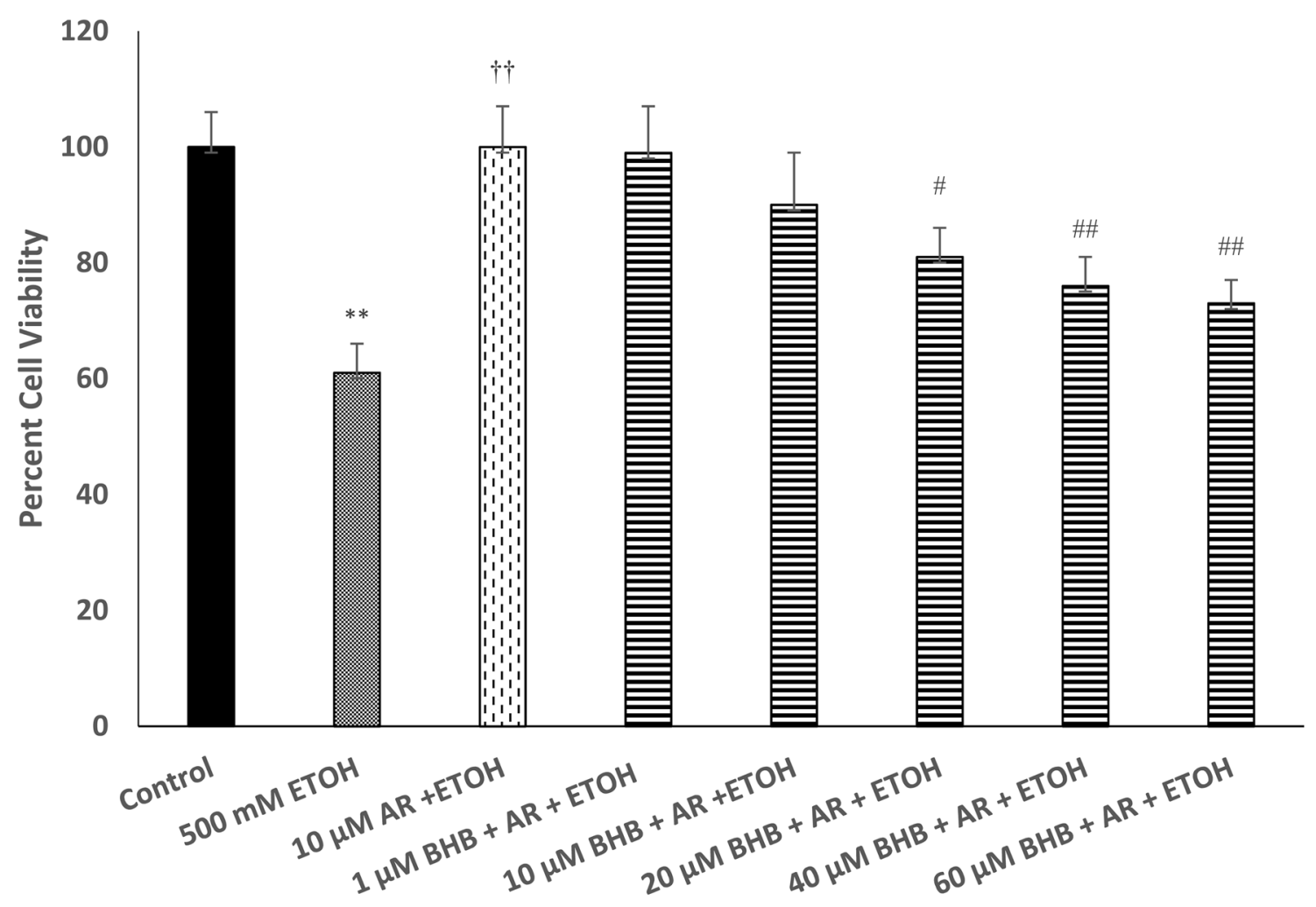

Fig. 4 Effect of various concentrations of beta-hydroxy butyrate (BHB), a FA3R antagonist, on protective effects of AR against ethanol (ETOH)-induced toxicity. Cells were treated with ETOH, AR, and BHB for $24 \mathrm{~h}$, and cell viability was determined by MTT. BHB

\section{Discussion}

The results of this study show that BUT as well as AR, an FA3R agonist, can protect against ETOH-induced toxicity in neuroblastoma-derived dopaminergic cells. Moreover, a synergistic protective effect of low concentrations of BUT and AR was also observed. Thus, a combination of ineffective or very low concentrations of BUT and AR resulted in more than an additive protection against ETOH toxicity. Pretreatment of cells with BHB, a selective FA3R antagonist, substantially reduced the protective effects of BUT and AR individually and completely blocked the protective effects of the combined concentrations of the two drugs. These results support potential beneficial effects of BUT and/or an FA3R agonist in alcohol toxicity.

The findings also extend the utility of short-chain fatty acids (SCFAs) beyond the neurodegenerative diseases such as Parkinson's disease (PD), to a common toxicant such as alcohol. Although further in vivo studies are required to validate this premise in relation to alcohol, such observations have been reported in PD models (St Laurent et al. 2013; Liu et al. 2017). Interestingly, in regard to cellular model of was added $1 \mathrm{~h}$ before AR, which was added $1 \mathrm{~h}$ before ETOH. Values are mean \pm SEM. ${ }^{*} p<<0.01$ compared to control. ${ }^{\dagger \dagger} p<0.01$ compared to ETOH, ${ }^{\#} p<0.05,{ }^{\# \#} p<0.01$ compared to AR + ETOH. $N=4$ per treatment

PD, protective effects of BUT against salsolinol were also partially blocked by $\mathrm{BHB}$, whereas the protective effects of AR against salsolinol were fully blocked by BHB (Getachew et al. 2020). Since AR is considered a full FA3R agonist (Hudson et al. 2014; Bolognini et al. 2016; Kaji et al. 2018) and BHB a selective FA3R antagonist (Kimura et al. 2011; Ulven 2012; Inoue et al. 2014), it was suggested that protective effects of BUT against salsolinol may involve more than FA3R activation (Getachew et al. 2020). This may include HDAC inhibition, activation of Nrf2/HO- 1 axis, and stimulation of glucagon like peptide-1 (Funakohi-Tago et al. 2018; Liu et al. 2017; Cantu-Jungles et al. 2019). Although it remains to be determined whether AR also shares any of such BUT characteristics, the finding that BHB could only partially block the protective effects of either BUT or AR against ETOH may also indicate involvement of other mechanism(s) in protection by BUT and AR against ETOH toxicity. However, the finding that the synergistic effects of the combination of BUT and AR could be fully blocked by BHB suggests that adequate activation of the FA3R might be sufficient for observed protection against ETOH toxicity and that the efficacy or potency of BHB to block FA3R might be limited. 


\section{BHB block of BUT+ AR protection against ETOH toxicity}

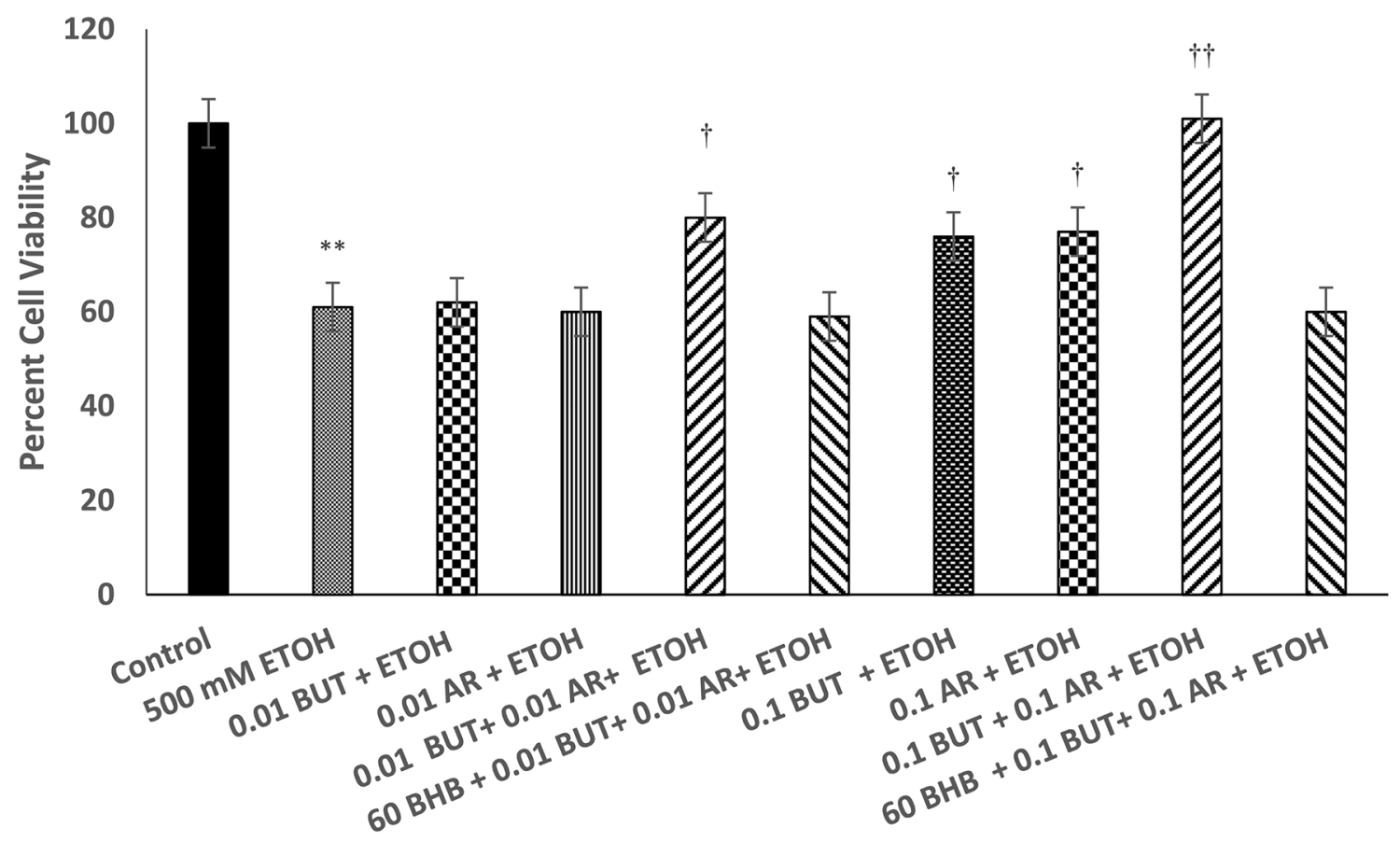

$\mu M A R, B U T$ \& BHB

Fig. 5 Effect of beta-hydroxy butyrate (BHB), a FA3R antagonist, on protective effects of the combination of butyrate (BUT) and AR against ethanol (ETOH)-induced toxicity. Cells were treated with $\mathrm{ETOH}, \mathrm{BUT}+\mathrm{AR}$, and BHB for $24 \mathrm{~h}$, and cell viability was deter-

SCFAs in general and BUT in particular may not only prevent neurotoxicity induced by such compounds as salsolinol or ETOH but may also be of potential utility in other diseases such as graft rejection, inflammatory bowel disease, colorectal cancer, and diabetes, all of which carry an inflammatory component (Tikhonova 2017; Alrafas et al. 2019; Baxter et al. 2019). As mentioned earlier, antiinflammatory effects of BUT are well established (CantuJungles et al. 2019). Interestingly, BUT may also be of therapeutic potential in both alcoholic- and non-alcoholic fatty liver diseases (Ralli et al. 2021; Zhang et al. 2021). In addition, BUT has been advocated for treatment of obesity and sleep disorders (Baxter et al. 2019; Szentirmai et al. 2019) and most recently for control of cytokine storm associated with COVID-19 (Nithin et al. 2021). However, because BUT has short half-life due to first-pass hepatic clearance (Miller et al. 1987; Kumar et al. 2019; Dingeo et al. 2020), AR, or any selective FA3R agonist, with a better pharmacologic profile than BUT, may offer a desirable alternative.

In summary, the findings support the potential use of butyrate and/or a selective FA3R agonist in combating alcohol toxicity. mined by MTT. BHB was added $1 \mathrm{~h}$ before BUT + AR, which was added $1 \mathrm{~h}$ before ETOH. Values are mean \pm SEM. ${ }^{* *} p<0.01 \mathrm{com}-$ pared to control. ${ }^{\dagger} p<0.5,{ }^{\dagger \dagger} p<0.01$ compared to ETOH. $N=4-5$ per treatment

Funding Supported by NIH/NIAAA R03AA022479 and Howard University College of Medicine Bridge Funds and Pilot Study Awards Program (BFPSAP) 2020-2021 (YT, RLC), NIA/NIH 1R25AG047843-01 (ABC).

\section{Declarations}

Conflict of Interest The authors declare no competing interests.

Disclaimers The authors indicate that the views expressed in the submitted article are their own and not an official position of the institution or the funding agency.

\section{References}

Alrafas HR, Busbee PB, Nagarkatti M, Nagarkatti PS (2019) Resveratrol modulates the gut microbiota to prevent murine colitis development through induction of Tregs and suppression of Th17 cells. J Leukoc Biol 106(2):467-480

Bassareo V, Frau R, Maccioni R, Caboni P, Manis C et al (2021) Ethanol-dependent synthesis of salsolinol in the posterior ventral tegmental area as key mechanism of ethanol's action on mesolimbic dopamine. Front Neurosci 15:675061

Baxter NT, Schmidt AW, Venkataraman A, Kim KS, Waldron C et al (2019) Dynamics of human gut microbiota and short-chain fatty 
acids in response to dietary interventions with three fermentable fibers. mBio.10(1):e02566-18

Belizário JE, Faintuch J (2018) Microbiome and Gut Dysbiosis Exp Suppl 109:459-476

Bishehsari F, Magno E, Swanson G, Desai V, Voigt RM et al (2017) Alcohol and Gut-Derived Inflammation Alcohol Res 38(2):163-171

Bolognini D, Tobin AB, Milligan G, Moss CE (2016) The pharmacology and function of receptors for short-chain fatty acids. Mol Pharmacol 89(3):388-398

Cantu-Jungles TM, Rasmussen HE, Hamaker BR (2019) Potential of prebiotic butyrogenic fibers in Parkinson's disease. Front Neurol 10:663

Carbia C, Lannoy S, Maurage P, López-Caneda E, O'Riordan KJ et al (2021) A biological framework for emotional dysregulation in alcohol misuse: from gut to brain. Mol Psychiatry 26(4):1098-1118

Cowan CSM, Cryan JF (2021) The microbiome-gut-brain axis in neurocognitive development and decline. Mod Trends Psychiatry $32: 12-25$

Cryan JF, O'Riordan KJ, Cowan CSM, Sandhu KV, Bastiaanssen TFS et al (2019) The microbiota-gut-brain axis. Physiol Rev 99(4):1877-2013

Dingeo G, Brito A, Samouda H, Iddir M, La Frano MR et al (2020) Phytochemicals as modifiers of gut microbial communities. Food Funct 11(10):8444-8471

Engen PA, Green SJ, Voigt RM, Forsyth CB, Keshavarzian A (2015) The gastrointestinal microbiome: alcohol effects on the composition of intestinal microbiota. Alcohol Res 37(2):223-236

Falomir-Lockhart LJ, Cavazzutti GF, Giménez E, Toscani AM (2019) Fatty acid signaling mechanisms in neural cells: fatty acid receptors. Front Cell Neurosci 13:162

Funakohi-Tago M, Sakata T, Fujiwara S, Sakakura A, Sugai T et al (2018) Hydroxytyrosol butyrate inhibits 6-OHDA-induced apoptosis through activation of the Nrf2/HO-1 axis in SH-SY5Y cells. Eur J Pharmacol 834:246-256

García-Cabrerizo R, Carbia C, Riordan O, KJ, Schellekens H, Cryan JF, (2021) Microbiota-gut-brain axis as a regulator of reward processes. J Neurochem 157(5):1495-1524

Getachew B, Csoka AB, Bhatti AC, RL, Tizabi Y, (2020) Butyrate protects against salsolinol-induced toxicity in SH-SY5Y cells: implication for Parkinson's disease. Neurotox Res 38:596-602

Getachew B, Hudson T, Heinbockel T, Csoka AB, Tizabi Y (2018) Protective effects of donepezil against alcohol-induced toxicity in cell culture: role of caspase-3. Neurotox Res 34(3):757-762

Giovannini MG, Lana D, Traini C, Vannucchi MG (2021) The microbiota-gut-brain axis and Alzheimer disease. From dysbiosis to neurodegeneration: focus on the central nervous system glial cells. J Clin Med 10(11):2358

Gupta H, Suk KT, Kim DJ (2021) Gut microbiota at the intersection of alcohol, brain, and the liver. J Clin Med 10(3):541

Hudson BD, Christiansen E, Murdoch H, Jenkins L, Hansen AH et al (2014) Complex pharmacology of novel allosteric free fatty acid 3 receptor ligands. Mol Pharmacol 86(2):200-210

Inoue D, Tsujimoto G, Kimura I (2014) Regulation of energy homeostasis by GPR41. Front Endocrinol (lausanne) 5:1-3

Jiang Y, Li K, Li X, Xu L, Yang Z (2021) Sodium butyrate ameliorates the impairment of synaptic plasticity by inhibiting the neuroinflammation in 5XFAD mice. Chem Biol Interact 341:109452

Kaji I, Akiba Y, Furuyama T, Adelson DW, Iwamoto K et al (2018) Free fatty acid receptor 3 activation suppresses neurogenic motility in rat proximal colon. Neurogastroenterol Motil 30(1): 10

Kelly DL, Kane MA, Fraser CM, Sayer MA, Grant-Beurmann S et al (2021) Prebiotic treatment increases serum butyrate in people with schizophrenia: results of an open-label inpatient pilot clinical trial. J Clin Psychopharmacol 41(2):200-202

Kimura I, Inoue D, Maeda T, Hara T, Ichimura A et al (2011) Shortchain fatty acids and ketones directly regulate sympathetic nervous system via G protein-coupled receptor 41 (GPR41). Proc Natl Acad Sci U S A 108:8030-8510

Knudsen JK, Bundgaard-Nielsen C, Hjerrild S, Nielsen RE, Leutscher $P$ et al (2021) Gut microbiota variations in patients diagnosed with major depressive disorder-a systematic review. Brain Behav e02177

Kong Q, Tian P, Zhao J, Zhang H, Wang G et al (2021) The autistic-like behaviors development during weaning and sexual maturation in VPA-induced autistic-like rats is accompanied by gut microbiota dysbiosis. PeerJ 9:e11103

Koob GF (2021) Drug addiction: hyperkatifeia/negative reinforcement as a framework for medications development. Pharmacol Rev 73(1):163-201

Kumar Singh A, Cabral C, Kumar R, Ganguly R, Kumar Rana H et al (2019) Beneficial effects of dietary polyphenols on gut microbiota and strategies to improve delivery efficiency. Nutrients 11(9):2216

Li H, Ni J, Hong Q (2021) Gut microbiota: critical controller and intervention target in brain aging and cognitive impairment. Frontiers in Aging Neuroscience 13:279

Liu J, Wang F, Liu S, Du J, Hu X et al (2017) Sodium butyrate exerts protective effect against Parkinson's disease in mice via stimulation of glucagon like peptide-1. J Neurol Sci 381:176-181

Lowe PP, Gyongyosi B, Satishchandran A, Iracheta-Vellve A, Cho $\mathrm{Y}$ et al (2018) Reduced gut microbiome protects from alcoholinduced neuroinflammation and alters intestinal and brain inflammasome expression. J Neuroinflammation 15(1):298

Meckel KR, Kiraly DD (2019) A potential role for the gut microbiome in substance use disorders. Psychopharmacology 236(5):1513-1530

Miller AA, Kurschel E, Osieka R, Schmidt CG (1987) Clinical pharmacology of sodium butyrate in patients with acute leukemia. Eur J Cancer Clin Oncol 23(9):1283-1287

Nithin KK, Patil P, Bhandary SK, Haridas V, Kumari SN et al (2021) Is butyrate a natural alternative to dexamethasone in the management of CoVID-19? F1000Res 10:273

Poluektova E, Yunes R, Danilenko V (2021) The putative antidepressant mechanisms of probiotic bacteria: relevant genes and proteins. Nutrients 13(5):1591

Ralli T, Neupane YR, Saifi Z, Kohli K (2021) Gut microbiota as an emerging therapeutic avenue for the treatment of non-alcoholic fatty liver disease. Curr Pharm Des. Epub ahead of print

Rodriguez-Gonzalez A, Orio L (2020) Microbiota and alcohol use disorder: are psychobiotics a novel therapeutic strategy? Curr Pharm Des 26(20):2426-2437

Romaní-Pérez M, Bullich-Vilarrubias C, López-Almela I, LiébanaGarcía R, Olivares M et al (2021) The microbiota and the gutbrain axis in controlling food intake and energy homeostasis. Int J Mol Sci 22(11):5830

St Laurent R, O’Brien LM, Ahmad ST (2013) Sodium butyrate improves locomotor impairment and early mortality in a rotenoneinduced Drosophila model of Parkinson's disease. Neuroscience 246:382-390

Szentirmai É, Millican NS, Massie AR, Kapás L (2019) Butyrate, a metabolite of intestinal bacteria, enhances sleep. Sci Rep 9(1):7035

Tikhonova IG (2017) Application of GPCR structures for modelling of free fatty acid receptors. Handb Exp Pharmacol 236:57-77

Tizabi Y, Getachew B, Aschner M (2021a) Novel pharmacotherapies in Parkinson's disease. Neurotox Res 39(4):1381-1390

Tizabi Y, Getachew B, Collins MA (2021b) Ethanol neurotoxicity. In: Kostrzewa RM (ed) Handbook of neurotoxicity, 2nd ed. Springer, Cham. https://doi.org/10.1007/978-3-030-71519-9_205-1 
Ullah H, Tovchiga O, Daglia M, Khan H (2021) Modulating gut microbiota: an emerging approach in the prevention and treatment of multiple sclerosis. Curr Neuropharmacol. Epub ahead of print

Ulven T (2012) Short-chain free fatty acid receptors FFA2/GPR43 and FFA3/GPR41 as new potential therapeutic targets. Front Endocrinol (lausanne) 3:111

van Krimpen SJ, Jansen FAC, Ottenheim VL, Belzer C, van der Ende $\mathrm{M}$ et al (2021) The effects of pro-, pre-, and synbiotics on muscle wasting, a systematic review-gut permeability as potential treatment target. Nutrients 13(4):1115

Woźniak D, Cichy W, Przysławski J, Drzymała-Czyż S (2021) The role of microbiota and enteroendocrine cells in maintaining homeostasis in the human digestive tract. Adv Med Sci 66(2):284-292
Yamashiro K, Kurita N, Urabe T, Hattori N (2021) Role of the gut microbiota in stroke pathogenesis and potential therapeutic implications. Ann Nutr Metab 9:1-9

Zhang T, Li J, Liu CP, Guo M, Gao CL et al (2021) Butyrate ameliorates alcoholic fatty liver disease via reducing endotoxemia and inhibiting liver gasdermin D-mediated pyroptosis. Ann Transl Med 9(10):873

Publisher's Note Springer Nature remains neutral with regard to jurisdictional claims in published maps and institutional affiliations. 